\title{
Steps Towards Developing a Cure for Autoimmunity
}

\author{
Tamara Zemlo \\ The Science Advisory Board, 2111 Wilson Boulevard, Suite 250, Arlington, VA 22201, USA
}

\begin{abstract}
Research into the molecular and cellular underpinnings of autoimmunity advances our understanding of immune functions and the role inflammation plays in mediating many of the symptoms of the multitude of autoimmune diseases. This knowledge serves as the foundation for identifying potential targets for treatment and developing novel therapies to prevent, modulate, and ultimately cure autoimmune diseases. The Science Advisory Board, in conjunction with BioInformatics, LLC (Arlington, Va), has interviewed hundreds of immunologists regarding the nature of their research and the scientific challenges they face in autoimmune disease research. Highlights from this benchmarking study are presented below in the context of promising research opportunities.
\end{abstract}

\section{Removing the barriers}

Hindered by technology barriers, research bottlenecks, and financial and time constraints, few pharmaceutical companies have successfully produced new therapeutics for autoimmune diseases in recent years. Improving this drug discovery process begins by first developing the underlying technologies that facilitate basic research science. Improved culturing techniques are needed to propagate human cells in the laboratory so that researchers may study how cells from diseased patients differ from cells from healthy individuals. Secondly, the discovery of new targets for autoimmune diseases is often complicated by the heterogeneity of autoimmune diseases. One way around this problem may be to focus on obtaining a more complete understanding of the genetics of autoimmune diseases, which scientists have identified as an immediate priority.

Correspondence and reprint requests to Tamara Zemlo, The Science Advisory Board, 2111 Wilson Boulevard, Suite 250, Arlington, VA 22201, USA, E-mail: t.zemlo@scienceboard.net

This is an open access article distributed under the Creative Commons Attribution License which permits unrestricted use, distribution, and reproduction in any medium, provided the original work is properly cited.
Once genes have been linked to specific autoimmune diseases, they can be categorized into groups based on their role in disease onset and progression. Genes that are associated with multiple diseases would make the most desirable targets for future therapeutics. Finally, the drug discovery and development process is often hampered by financial and time constraints. By forming collaborations with outside researchers, pharmaceutical and biotechnological companies gain access to cutting-edge research without spending the time and resources needed to create specialized, autoimmunity R \& D programs in-house. The results of this research can serve as a sturdy foundation that supports industry's future drug discovery efforts dedicated to inventing novel autoimmune therapies.

\section{Harnessing the power of genetics}

The majority of immunologists interviewed assert that the research breakthrough that has the best hope of leading to effective treatments for autoimmune diseases is the identification of genes whose altered activity might contribute to autoimmunity. Furthermore, enhancing these correlations with population studies that reveal polymorphisms and linking these genes with a predisposition to a particular disease or constellation of symptoms will be necessary in order to develop new therapies and treatments within the next decade.

As scientists strive to uncover critical genetic linkages, they continue to develop a greater understanding of the molecular basis of these diseases. Despite this increasing comprehension, research efforts to date have been broad and involve numerous approaches because autoimmune diseases encompass a diverse set of molecular components and clinical events. However, recent advances in molecular biology techniques have enabled the discovery of specific autoantigens implicated in a variety of autoimmune disorders. Knowledge of the molecular pathways controlling the production of these autoantigens has enabled the pharmaceutical and biotechnology industry to focus on the triggers and target molecules involved in causing autoimmunity.

With the ability to pinpoint genetic defects contributing to autoimmune diseases, more and more emphasis 
is being placed on genetic therapy, especially an ex vivo approach. Antigen-specific $\mathrm{CD}^{+} \mathrm{T}$ cells and antigenpresenting dendritic cells are critical mediators in the pathogenesis of autoimmune diseases and are proving to be ideal candidates for adoptive cellular gene therapy.

\section{The quest to control cell signaling}

Notwithstanding the excitement surrounding genetic therapies, most researchers interviewed indicated that cell signaling regulators hold the most promise for treating autoimmune diseases within the next decade. In an attempt to find new regulators of the immune system, researchers are utilizing functional genome-wide screens, which allows for the functional cloning of regulators involved in multiple signal transduction pathways. A main attraction of this strategy for the pharmaceutical and biotechnology industry is that it requires no prior sequence information; and it does not skew the search to previously known signaling molecules. The absence of these technical limitations opens up the possibility for discovering many novel targets to control the immune system through cell signaling pathways.

\section{Strategies for tolerance induction}

Ideally it would be most effective to prevent the loss of immunological tolerance instead of trying to arrest the progress of autoimmunity. However, identifying those individuals who are at risk for a wide range of autoimmune diseases is a daunting epidemiologic prospect. While some researchers are concentrating on this challenge, treatment breakthroughs for those individuals who already have an autoimmune disease have evolved from interventions based upon immunosuppression of those built upon immunomodulation.

By devoting their $\mathrm{R} \& \mathrm{D}$ efforts to refining the mechanism of immunomodulation, pharmaceutical and biotechnology companies will be able to devise even more effective therapies. There is increasing interest in the use of stem cell therapeutics to accomplish this goal. The majority of scientists questioned, however, believe that characterizing the mechanism of tolerance breakdown contributed by $\mathrm{CD} 4{ }^{+} \mathrm{CD} 25^{+}$regulatory $\mathrm{T}$ cells in association with NKT cells will most aid in the advancement of autoimmune disease research.

\section{Profiling future progress}

The pharmaceutical and biotechnology industry should place a continued emphasis on identifying putative autoantigens by applying more and more sophisticated functional genomics techniques. Knowledge gained from these experiments will likely be used to delve deeper into the genetic, environmental, and infectious causes of certain autoimmune diseases. This focus will include efforts to explain the gender differences that are observed in susceptibility to autoimmune diseases including, but are not limited to, hormonal differences.
Further into the future, a variety of genetic therapy approaches will become increasingly vital in treating autoimmune diseases through the transfer of genes encoding for immunomodulatory cytokines or antigens that would induce tolerization. Emphasis should thus be placed on better understanding the heterogeneity of autoimmune diseases in order to simplify the search for these common pathway targets.

Although progress in autoimmunity typically occurs by building upon a solid body of research, scientists must learn to adapt and modify their objectives to incorporate innovative hypotheses. Whether or not new research findings result in a major paradigm shift in our understanding of the fundamental causes of autoimmunity, they do reinforce the certainty that it is only by basing strategic R \& D plans on basic research insights that substantial progress can be made in developing the next generation of tools that will enable the discovery of valuable therapeuticsand even a cure for autoimmune diseases.

Tamara Zemlo

Tamara Zemlo, who holds the $\mathrm{PhD}$ and MPH degrees, is the Director of Scientific and Medical Communications for The Science Advisory Board, Division of BioInformatics LLC, a life science market research and consulting firm located in Arlington, Virginia. The Science Advisory Board is an international community of more than 25000 life science and medical professionals. Board members convene to participate

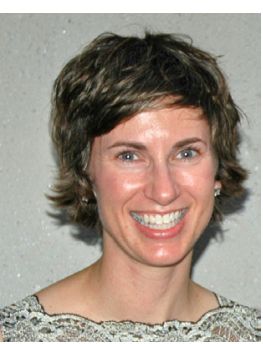
in online conferences, surveys, and discussions addressing issues of importance to their individual areas of investigation and/or clinical specialties. 


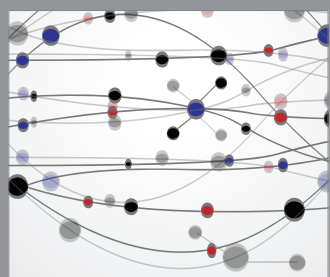

The Scientific World Journal
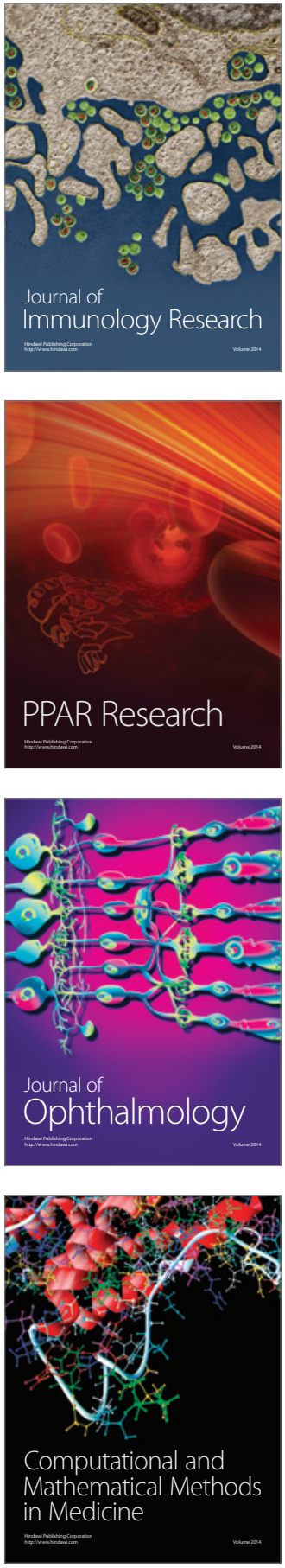

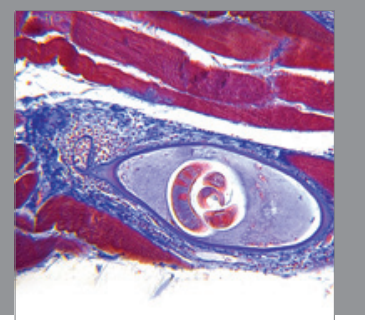

Gastroenterology

Research and Practice
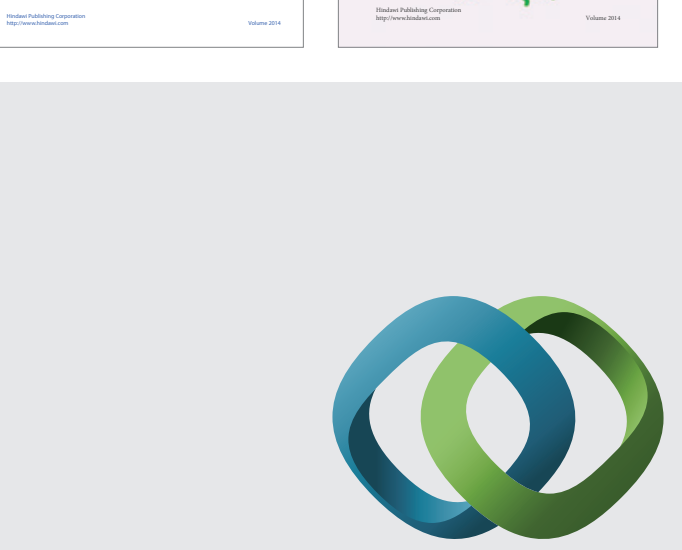

\section{Hindawi}

Submit your manuscripts at

http://www.hindawi.com
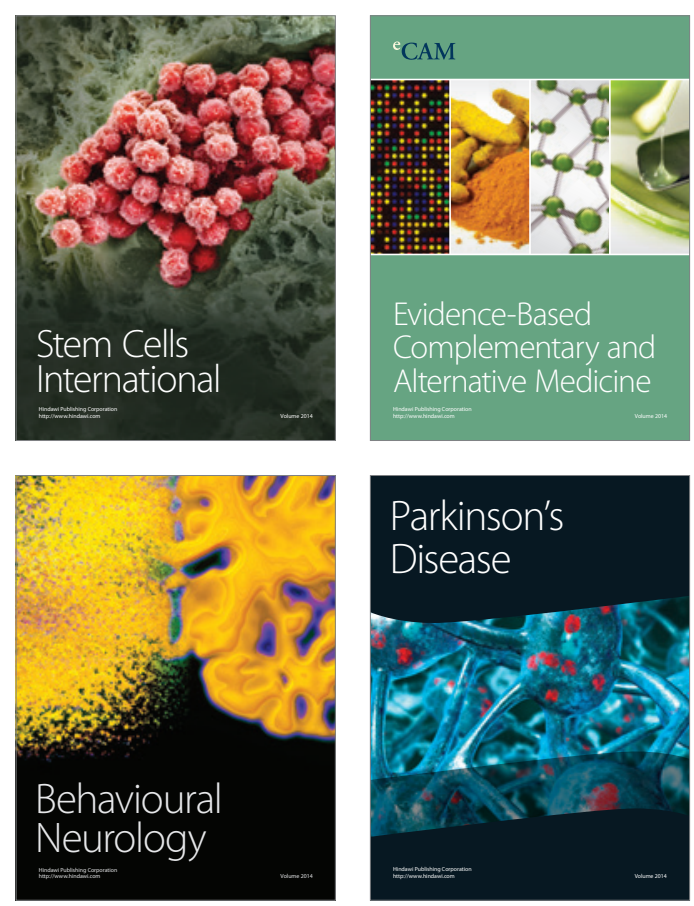

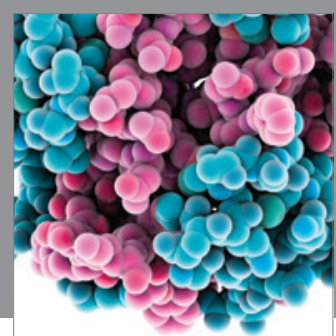

Journal of
Diabetes Research

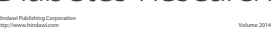

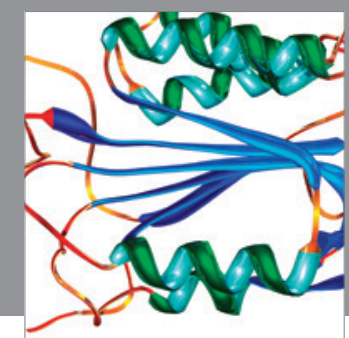

Disease Markers
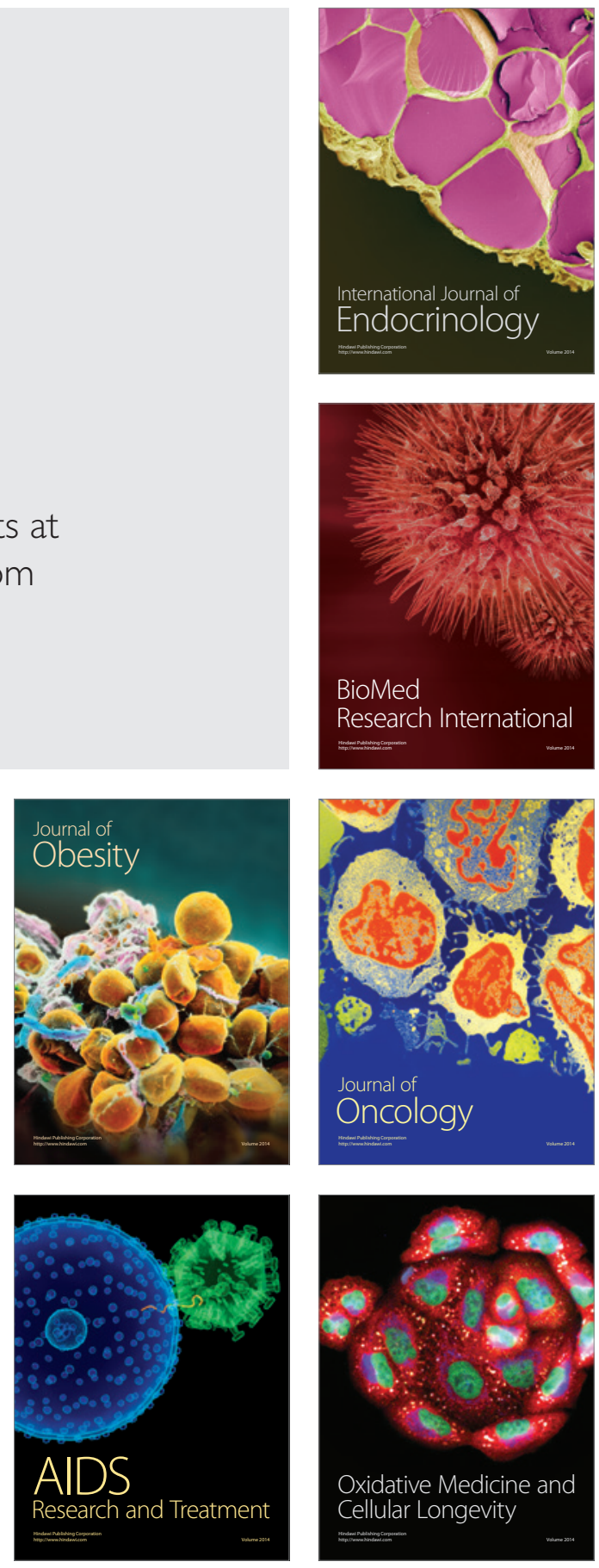